\title{
Association between tumor location and neurocognitive functioning using tumor localization maps
}

Esther J.J. Habets ${ }^{1 *}$ MSc, Eef J. Hendriks ${ }^{2 *}$ MD, Martin J.B. Taphoorn ${ }^{1,3}$ MD, Linda Douw ${ }^{4,5}$ PhD, Aeilko H. Zwinderman ${ }^{6}$ MD, W. Peter Vandertop ${ }^{7}$ MD, Frederik Barkhof ${ }^{2,8}$ MD, Philip C. De Witt Hamer ${ }^{7}$ MD, Martin Klein ${ }^{9} \mathrm{PhD}$

${ }^{1}$ Department of Neurology, Haaglanden Medical Center, PO BOX 432, 2501 CK, The Hague, The Netherlands;

${ }^{2}$ Department of Radiology \& Nuclear Medicine, Amsterdam University Medical Center, location VUmc, De Boelelaan 1118, 1081 HZ, Amsterdam, The Netherlands;

${ }^{3}$ Department of Neurology, Leiden University Medical Center, PO BOX 9600, 2300 RC, Leiden, The Netherlands;

${ }^{4}$ Department of Anatomy and Neurosciences, Amsterdam University Medical Center, location VUmc, De Boelelaan 1118, 1081 HZ, Amsterdam, The Netherlands;

${ }^{5}$ Department of Radiology, Athinoula Martinos Center for Biomedical Imaging, Massachusetts General Hospital, 149 Thirteenth Street, Charlestown, Massachusetts, US;

${ }^{6}$ Department of Clinical Epidemiology and Biostatistics, Amsterdam University Medical Center, location AMC, Meibergdreef 9, 1105 AZ, Amsterdam, The Netherlands;

${ }^{7}$ Neurosurgical Center Amsterdam, Amsterdam University Medical Center, De Boelelaan 1117, 1081 HV, Amsterdam, The Netherlands;

${ }^{8}$ Institutes of Neurology \& Healthcare Engineering, UCL, London, UK

${ }^{9}$ Department of Medical Psychology and Brain Tumor Center, Amsterdam University Medical Center, location VUmc, De Boelelaan 1118, 1081 HZ, Amsterdam, The Netherlands.

* These authors contributed equally

Contact corresponding author:

E.J.J. Habets

Department of neurology

Haaglanden Medical Centre

Lijnbaan 32

PO Box 432

2501 CK The Hague 
The Netherlands

e-mail: e.habets@haaglandenmc.nl

Telephone number: +31(00)088-9792495

ORCID corresponding author: $\underline{0000-0001-5938-9792}$

Word count abstract: 249

Word count: 2995

Number of references: 50

Number of tables and figures: 5

There is electronic supplementary material to the manuscript

Key words: glioma, neurocognitive functioning, MRI, functional mapping

\section{Acknowledgements}

This study is part of the program Innovative Medical Devices Initiative with project number 10-

10400-96-14003, which is financed by the Netherlands Organization for Scientific Research (NWO). This research is also supported by a research grant from the Dutch Cancer Society (VU2014-7113). 


\begin{abstract}
Introduction: Patients with diffuse glioma often experience neurocognitive impairment already prior to surgery. Pertinent information on whether damage to a specific brain region due to tumor activity results in neurocognitive impairment or not, is relevant in clinical decision-making, and at the same time renders unique information on brain lesion location and functioning relationships. To examine the impact of tumor location on preoperative neurocognitive functioning (NCF), we performed MRI based lesion-symptom mapping.
\end{abstract}

Methods: Seventy-two patients (mean age 40 years) with a radiologically suspected glioma were recruited preoperatively. For each of the six cognitive domains tested, we used tumor localization maps and voxel-based lesion-symptom mapping analyses to identify cortical and subcortical regions associated with NCF impairment.

Results: Compared to healthy controls, preoperative NCF was significantly impaired in all cognitive domains. Most frequently affected were attention (30\% of patients) and working memory (20\% of patients). Deficits in attention were significantly associated with regions in the left frontal and parietal cortex, including the precentral and parietal-opercular cortex, and in left-sided subcortical fiber tracts, including the arcuate fasciculus and corticospinal tract. Surprisingly, no regions could be related to working memory capacity. For the other neurocognitive domains, impairments were mainly associated with regions in the left hemisphere.

Conclusions: Prior to treatment, patients with diffuse glioma in the left hemisphere run the highest risk to have NCF deficits. Identification of a left frontoparietal network involved in NCF not only may optimize surgical procedures but may also be integrated in counseling and cognitive rehabilitation for these patients. 


\section{INTRODUCTION}

While patients with gliomas often experience impairment in physical and emotional functioning, specifically deficits in neurocognitive functioning (NCF) negatively affect daily life functioning and health-related quality of life [1]. These deficits can be caused by the tumor and surrounding edema, by epilepsy and anti-epileptic drugs, and by tumor treatment, including surgery, radiotherapy and chemotherapy [1].

There is substantial evidence that the tumor itself is the major cause of neurocognitive deficits in glioma patients. Prior to treatment, impairment in working memory [2], memory [3], executive functioning [3, 4], and language capacity [4, 5] have been observed.

Regarding surgery, usually the first step in treatment of these patients, detailed knowledge on the neuroanatomy-function associations is pivotal to attain an optimal balance between tumor control (e.g. OS) by resection and functional outcome (e.g. NCF). Although available data on the involvement of specific brain regions involved in language [5], and verbal or perceptual abilities [6] certainly are of value, more detailed information regarding the involvement of specific cortical or subcortical brain regions and tracts on NCF is needed. To identify those brain regions critically involved in NCF, voxelbased lesion-symptom mapping [7] is a promising tool that is well-established in stroke [8] and traumatic brain injury $[9,10]$. However, in brain tumor patients voxel-based lesion-symptom mapping has been applied in only a few postoperative $[11,12]$ and preoperative [13] studies, with a focus on one specific neurocognitive function or specific brain regions. The use of so-called tumor localization maps (TLMs) in order to voxel-wise link brain tumor location to NCF, can add pertinent information to the associations known from non-tumor lesion studies, as gliomas have differences in preference location, nature and infiltrative growth pattern compared to other, acute, lesions [13]. Voxel-by-voxel lesion mapping has the advantage that a priori lesion location grouping is avoided [7, 14] and that white matter structures can additionally be identified.

The aim of the present study is to better understand the impact of brain tumor locations on distinct neurocognitive functions in glioma patients prior to surgery, by using voxel-based lesion-function mapping.

\section{METHODS}

\section{Patients}

A retrospective observational cohort of consecutive de novo patients planned to undergo resective surgery for suspected glioma in Amsterdam UMC, a tertiary referral center for neuro-oncological surgery, was established. Adult patients ( $\geq 18$ years) were included if they had a radiologically suspected glioma, no prior surgery, no radiotherapy or chemotherapy, preoperative neurocognitive assessment, and preoperative MRI including 3D FLAIR and 3D T1 gadolinium. Neuropsychological assessment and MRI were part of routine clinical care. Exclusion criteria were history of neurological 
or severe psychiatric disorder potentially interfering with NCF, and insufficient command of the Dutch language.

\section{Standard protocol approvals and patient consent}

Approval of the study protocol by the institutional review board of the Amsterdam UMC was not required according to the Dutch law on Medical Research in Humans. All patients gave written informed consent for using clinically derived data, including neuropsychological and MRI data, for research purposes.

\section{Neurocognitive assessment}

NCF was assessed by a battery of standardized neuropsychological tests, covering the range of neurocognitive functions known to be affected in glioma patients $[6,15]$. We combined test outcomes into six cognitive domain scores, based on previous studies and consensus in neuropsychological practice: information processing speed, attention, working memory, verbal memory, visual memory and executive functioning (Online Resource 1) [15, 16].

For each test, the patient's performance was compared to published normative data of healthy controls, corrected for age and educational level, or with performance of healthy controls from a database of the Maastricht Aging study, The Netherlands [17]. These controls where individually matched to patients according to age, gender, and educational level [18]. Individual patient test scores were converted into standardized z-scores with use of mean and standard deviation (SD) of the healthy controls on that test outcome. Domain summary measures were calculated for the patients, by dividing the total score of the test scores contributing to the domain, by the number of test outcomes of that domain. A domain score of $\geq 1.5$ SD below the mean of controls was considered a clinically significant neurocognitive impairment [19].

\section{MRI scanning}

All patients were scanned using a standardized neuro-oncology protocol. The protocol included a 3D FLAIR turbo spin-echo pulse-sequence and a 3D heavily T1-weighted gradient-echo pulse-sequence obtained after double-dose administration of intravenous gadolinium. Scans were available from various 1.5 and 3.0T scanners (including GE Signa HDXT, Toshiba Titan, Siemens Avanto, and Philips Ingenia). All MRI images were acquired with $\sim 1 \mathrm{~mm}$ isotropic resolution.

\section{Voxel-based lesion mapping}

For tumor localization mapping, we used the methodology described elsewhere [20]. Tumor regions were segmented in 3D on FLAIR images (smart brush tool of iPlan v3.0 software; BrainLAB AG, Feldkirchen, Germany) in case of a suspected low-grade glioma (i.e. hyperintense signal abnormality) and on $\mathrm{T} 1$ post contrast images in case of a high-grade glioma (i.e. contrast-enhancement 
abnormality). The segmented volumes were verified and adjusted in reconstruction planes by two observers (EH and PW). The FLAIR scans were linearly registered to the T1 scans for further analysis using the image fusion tool. Perilesional edema was excluded from the segmentations. Subsequently, the segmented volumes were exported as binary masks registered to the T1 images. The resulting patient-specific T1 volume was non-linearly registered to $2 \times 2 \times 2 \mathrm{~mm}$ Montreal Neurological Institute brain template (MNI-152 space), i.e. standard brain space [21]. This registration was done in sequential steps, with rigid, affine, B-spline regularization and symmetric diffeomorphic registration with cross-correlation as similarity metric [22]. The registration was visually verified and repeated when necessary. The results were preoperative TLMs of all patients grouped together, aligned to MNI space for further analysis. The probability of tumor was calculated for each voxel that covered MNI space, by dividing the number of patients with a glioma at that voxel by the total number of patients.

\section{Statistical analyses}

For a detailed description of the statistical analysis of the TLMs we refer to our previous publication [20]. In short, to identify brain regions associated with cognitive impairment, for each neurocognitive domain we compared TLMs of patients with and without neurocognitive impairment in the presence of a glioma at a specific voxel using a voxel-wise randomization test. We restricted the analyses to brain regions affected by tumor in at least three patients. As test statistic per voxel, the fraction of patients with a tumor region among those with an impairment was divided by the fraction of patients with a tumor region among those without, providing a 'relative risk'. A higher test statistic corresponded to a higher rate of tumor location regions among patients with an impairment than among those without.

To evaluate the probability of the test statistics, we used a randomization test. The spatial dependency of the data was addressed by estimating the empirical null-distribution of relative risks for each voxel based on a permutation with relabeling of patients to one of the two cohorts. A subset of 4,000 randomizations was randomly drawn without replacement to provide a reasonable estimate, as has been applied to analyze functional neuroimaging [23-25]. The adjusted one-sided p-value per voxel was calculated by counting the number of equal or more extreme test statistics in the randomized null distribution, and dividing by the number of randomizations. Multiple hypothesis testing was controlled for type I error using the false discovery rate, expressed as q-value for each voxel. These q-values are the proportion of voxels with false differential probability of a glioma among all voxels declared differentially infiltrated, when the p-value of a voxel is called significant. To obtain the q-value for a voxel, we divided the estimated number of false discoveries by the number of voxels declared significant. The estimated number of false discoveries given a specific adjusted p-value threshold was determined from the empirical null-distribution of 4,000 randomizations per voxel. The number of voxels declared significant was determined from the distribution of observed p-values [23, 25]. This resulted in p- and q-value maps of differentially infiltrated brain regions that were superimposed on 
MNI-152 space for visual inspection. Because of the exploratory nature of our study, we considered $\mathrm{q}<0.4$ as informative, and $\mathrm{q}<0.2$ as significant. The correlation between the number of tumor voxels and the neurocognitive domain score (impaired versus not impaired) was analyzed using a nonparametric Spearman test. For further interpretation of the brain locations, we aligned our results with standard cortical [26] and white matter anatomy [27]. Statistical procedures were customized in R, v3.3 [28].

\section{Language functioning as reference measure}

To ensure the validity of our map comparisons, as the use of TLMs is a relatively new approach in glioma patients, we used language functioning as a reference measure. A map calculating the association between preoperative tumor location and language impairment was added. Language function was tested during preoperative neurological examination and was characterized mainly by naming and (subjective) word finding problems.

\section{RESULTS}

\section{Sociodemographic and clinical data}

Seventy-two patients were included (table 1). Excluded were ten cases: three patients with incomplete neuropsychological assessment, two patients with a history of previous surgery, one patient with both previous surgery and radiotherapy, two patients with a cavernous malformation, one patient with an epidermoid and one patient with a hypothalamus hamartoma. The majority (60\%) of patients, with a mean age of 40 years, was diagnosed with a glioma WHO grade II. Forty-one tumors were located in the left, and 31 in the right hemisphere. Epilepsy was the most frequently presenting symptom (62\%), in $10 \%$ of cases in combination with neurocognitive impairment. Tumors were predominantly located in frontal, temporal and insular brain regions (Figure 1). In two patients with right sided tumor and left handedness, additional fMRI revealed right hemispheric dominance for language functions. In two left handed patients with tumor in the left hemisphere, fMRI showed right hemisphere dominance for language. For all other patients, left hemisphere was language dominant. The mean interval between preoperative MRI and neurocognitive assessment was 0.4 weeks ( $\mathrm{SD} \pm 9.2$ weeks).

\section{Neurocognitive outcome}

Attention and working memory capacity were the most frequently affected domains, in $30 \%$ and $20 \%$ of patients respectively. Fewer patients experienced impaired visual memory (14\%), verbal memory (13\%), information processing speed (11\%), and executive functioning (8\%) (Figure 2).

\section{Brain regions associated with neurocognitive functions}

Reference measure: language functioning 
Only regions in the left hemisphere were found to be significantly associated with language functioning, including the frontal operculum, supra-marginal gyrus, arcuate fasciculus, superior- and middle frontal gyrus, superior temporal gyrus, and insular cortex (Online Resource 2). As the significant language regions and tracts in our sample are in accordance with earlier studies on naming and word retrieval processing in the brain $[29,30]$, tumor location mapping can be considered a valid method to link NCF to brain regions.

\section{Neurocognitive functions}

Attentional performance was significantly associated with regions of the left frontal and parietal cortex (Figure 3a; Online Resource 3), including precentral and postcentral gyrus, central and parietal operculum (Figure 4). Moreover, significant regions were observed in the insular cortex, Heschl's gyrus and the planum temporale, and in white matter tracts (arcuate fasciculus and corticospinal tract). In the right hemisphere, only a focal area of voxels in the parahippocampal gyrus exceeded the critical q-value.

For working memory, no significant clusters were identified (Figure 3b; Online Resource 4). P-values indicate bilateral parietal and frontal regions, predominantly in left hemisphere, possibly involved in working memory performance, but these were not identified in our q-value maps.

Visual memory, verbal memory, information processing speed and executive functioning were also analyzed for associated brain regions, although these were involved in fewer patients. Overall, for these four domains, regions in left white matter structures, including arcuate fasciculus, corticospinal tract, and corpus callosum, and in left frontal and parietal cortex, including pre- and postcentral gyrus, were found to be significantly associated with performance (Figure 4; Online Resource 5-8). In addition, visual and verbal memory impairments were associated with regions in the left cingulum, left posterior cingulate gyrus and precuneus. For verbal memory and information processing speed, the hippocampus showed significant voxels. Many other regions were identified to be significantly associated with executive functioning, most importantly the inferior fasciculus and superior parietal lobe. Right-sided regions, including temporal pole, fusiform cortex and basal ganglia, were only associated with verbal memory.

\section{DISCUSSION}

We aimed to better understand the association between tumor location and distinct neurocognitive functions in glioma patients prior to surgery. Attention and working memory were the most affected domains. Using voxel-based lesion mapping we observed brain structures associated with attention impairments to be localized in the left frontal and parietal cortex and left-sided white matter tracts. The neural basis for attentional functioning has been extensively studied. One of the most influential models is the three attentional networks model (alerting, orienting and executive control) [31, 32], which involves an extensive anatomical distribution including frontal and parietal regions with 
interconnecting pathways, the anterior cingulate cortex and dorsolateral prefrontal cortex, and the thalamus with connections to the superior parietal lobe, temporo-parietal junction, the superior temporal lobe, and the frontal eye fields $[31,33,34]$. Our results comply to some extent with this anatomical attentional network, i.e. we found significant regions in prefrontal and parietal areas. However, we could not replicate the involvement of the anterior cingulate cortex in executive control $[34,35]$, nor did we observe regions associated with the orienting and alerting networks of attention $[31,35]$. This could be due to an underrepresentation of gliomas in these regions or by the use of other attentional tasks than the ones used in Posner's model [34] that thus might have provoked activation in different brain regions. Contrasting with earlier studies, apart from the parahippocampal gyrus, we did not identify regions associated with aspects of attention in the right hemisphere [31, 36], including the inferior frontal gyrus and medial temporal cortex found in stroke patients [37] and the caudal anterior cingulate in traumatic brain injury patients [54]. Nor did we observe bilateral activation in the thalamus [35, 38] and basal ganglia [38]. Perhaps we did not find associated regions in the right hemisphere because our testing protocol consisted of tasks requiring a verbal response, and did not assess spatial attention.

Surprisingly, we also observed sections of the white matter tracts, especially the arcuate fasciculus and the corticospinal tract, to be involved in attentional functioning. The corticospinal tract is mainly involved in motor function, which was not required in the applied tasks. The arcuate fasciculus is known for its role in language processing [30], but scarce data also suggest a role in attentional processes and executive functioning, for example in patients with attention deficit hyperactivity disorder, using diffusion spectrum imaging tractography [39]. As the involvement of the arcuate fasciculus was also found for memory, speed and executive functioning, and most tasks were presented verbally and/or required a verbal response, glioma infiltrating in the arcuate fasciculus could have a subtle impact on neurocognitive performance.

No specific regions were identified to be associated with working memory, perhaps due to low statistical power. In the literature, many different structures within the prefrontal-parietal network have been associated with working memory performance in healthy and brain injured patients, partly depending on the type of working memory task. For example, impairments in complex span tasks were associated with lesions in the left inferior frontal gyrus [40]. Other relevant regions, found in fMRI studies, include the bilateral posterior parietal cortex [41, 42] premotor areas and posterior cingulate [41], insular cortex and middle temporal gyrus, the inferior longitudinal and fronto-occipital fasciculi, uncinate, and corpus callosum [42].

Our study evidently has some limitations. First, including low-grade glioma might have complicated the analysis, as these tumors do not show contrast enhancement on MRI and hence tumor boundaries are more difficult to identify. Also the behavior and biology of low- and high-grade glioma might affect the infiltration in brain tissue in a variable way [43], complicating lesion mapping and therefore, according to some authors, makes this method not applicable in glioma patients [44]. However, the 
tumor maps were also based on FLAIR images, and in accordance with others [13, 45] we are convinced that voxel-based lesion mapping is applicable in gliomas. Second, differences in the concept of brain plasticity might hamper interpretation of our results. For example, the higher growth rate associated with isocitrate dehydrogenase 1 wild type (IDH1-WT) tumors compared to IDH1mutant tumors, might result in less efficient brain networks and less time for brain plasticity to compensate for functional loss [46]. Also, functional compensation might be better for cognitive functions that are represented in more widespread brain networks, such as language [47], than for more basic functions such as spatial attention [13], although results in literature are partially contradictory [48]. As a result, we might have missed brain regions involved in specific neurocognitive functions seen in acute lesions. However, this does not negate that the regions we found to be associated with NCF are still of relevance. Third, due to unevenly distributed glioma over cortical and subcortical areas, not all regions could be included in our analysis. Therefore, perhaps not all brain structures relevant for the tested neurocognitive functions could be identified. Fourth, the interval between cognitive assessment and MRI scanning varied. In patients with a relatively long interval, the location, size and infiltration pattern of glioma tissue, as observed on MRI, could have been different from the glioma at time of the neurocognitive assessment. However, especially highgrade gliomas show rapid change and these patients were scanned within a relatively short interval. Fifth, we did not calculate Jaccard/Dice overlap scores between the two observers (EH and PW). The interrater agreement for tumor segmentation before surgery in general is known to be non-trivial [49]. Sixth, neuropsychological assessment was more routinely performed in WHO grade II glioma patients, who are typically young adults with an anticipated survival of many years. Cognitive performance is important for social and professional functioning during these years. For patients with WHO grade IV glioma, who still have a relatively short survival, extensive neuropsychological assessment was less well possible due to physical status or the necessity of planning the surgery at short notice. For this reason, high-grade glioma patients are underrepresented in our study cohort.

Finally, because of the explorative nature of our study design, we did not examine the influence of epilepsy, use of anti-epileptic drugs, emotional changes, depression, anxiety and fatigue on NCF, while these factors are relevant in glioma patients and have an impact on cognition [50, 51, 52, 53]. Our study has several implications. First, our finding regarding the involvement of the corticospinal tract in attention adds some information to the field of neuroscience. This observation should be confirmed in larger series. Second, as preoperative neurocognitive impairment in glioma patients is common, and mainly associated with disruption of the left frontoparietal network, assessment of attention and working memory prior to surgery, and informing patients regarding these impairments, should be incorporated in clinical care. Third, during awake surgical procedures resective surgery can be optimized by intraoperative monitoring of attentional function. However, rigorous paradigms to test these functions are yet to be developed. Finally, with information regarding preoperative 
neurocognitive impairment, cognitive rehabilitation can be applied in an early stage and tailored to the individual patient.

\section{Acknowledgements}

None.

\section{COMPLIANCE WITH ETHICAL STANDARDS}

\section{Funding}

This study is part of the program Innovative Medical Devices Initiative with project number 1010400-96-14003, which is financed by the Netherlands Organization for Scientific Research (NWO). This research is also supported by a research grant from the Dutch Cancer Society (VU2014-7113).

\section{Conflict of interest}

The authors declare that they have no conflict of interest.

\section{Ethical approval}

All procedures performed in studies involving human participants were in accordance with the ethical standards of the institutional and/or national research committee and with the 1964 Helsinki declaration and its later amendments or comparable ethical standards.

\section{Informed consent}

Informed consent was obtained from all individual participants included in the study.

\section{Electronic Supplementary Material}

Table 1, videos 1-7. 


\section{References}

1. Taphoorn MJ, Klein M (2004) Cognitive deficits in adult patients with brain tumours. Lancet Neurol 3(3):159-68.

2. Teixidor P, Gatignol P, Leroy M, Masuet-Aumatell C, Capelle L, Duffau H (2007) Assessment of verbal working memory before and after surgery for low-grade glioma. J Neurooncol 81(3):305-13.

3. Papagno C, Casarotti A, Comi A, Gallucci M, Riva M, Bello L (2012) Measuring clinical outcomes in neuro-oncology. A battery to evaluate low-grade gliomas (LGG). J Neurooncol 108(2):269-75.

4. Satoer D, Vork J, Visch-Brink E, Smits M, Dirven C, Vincent A (2012) Cognitive functioning early after surgery of gliomas in eloquent areas. J Neurosurg 117(5):831-8.

5. Duffau H, Moritz-Gasser S, Mandonnet E (2014) A re-examination of neural basis of language processing: proposal of a dynamic hodotopical model from data provided by brain stimulation mapping during picture naming. Brain Lang 131:1-10.

6. Klein M, Taphoorn MJ, Heimans JJ, van der Ploeg HM, Vandertop WP, Smit EF, et al (2001) Neurobehavioral status and health-related quality of life in newly diagnosed high-grade glioma patients. J Clin Oncol 19(20):4037-47.

7. Bates E, Wilson SM, Saygin AP, Dick F, Sereno MI, Knight RT, et al (2003) Voxel-based lesionsymptom mapping. Nat Neurosci 6(5):448-50.

8. Biesbroek JM, Kuijf HJ, van der Graaf Y, Vincken KL, Postma A, Mali WP, et al (2013) Association between subcortical vascular lesion location and cognition: a voxel-based and tract-based lesion-symptom mapping study. The SMART-MR study. PLoS One 8(4):e60541.

9. Perlbarg V, Puybasset L, Tollard E, Lehericy S, Benali H, Galanaud D (2009) Relation between brain lesion location and clinical outcome in patients with severe traumatic brain injury: a diffusion tensor imaging study using voxel-based approaches. Hum Brain Mapp 30(12):3924-33.

10. Barbey AK, Colom R, Solomon J, Krueger F, Forbes C, Grafman J (2012) An integrative architecture for general intelligence and executive function revealed by lesion mapping. Brain 135(Pt 4):1154-64. 
11. Campanella F, D'Agostini S, Skrap M, Shallice T (2010) Naming manipulable objects: anatomy of a category specific effect in left temporal tumours. Neuropsychologia 48(6):1583-97.

12. Mandonnet E, De Witt Hamer P, Poisson I, Whittle I, Bernat AL, Bresson D, et al (2015) Initial experience using awake surgery for glioma: oncological, functional, and employment outcomes in a consecutive series of 25 cases. Neurosurgery 76(4):382-9; discussion 9.

13. Shallice T, Mussoni A, D'Agostino S, Skrap M (2010) Right posterior cortical functions in a tumour patient series. Cortex 46(9):1178-88.

14. Rorden C, Karnath HO, Bonilha L (2007) Improving lesion-symptom mapping. J Cogn Neurosci 19(7):1081-8.

15. Meyers CA, Cantor SB (2003) Neuropsychological assessment and treatment of patients with malignant brain tumors. In: Prigatano GP, Pliskin NH (eds) Clinical neuropsychology and cost outcome research, a beginning. New York: Psychology Press, Inc pp 159-73.

16. Douw L, Klein M, Fagel SS, van den Heuvel J, Taphoorn MJ, Aaronson NK, et al (2009) Cognitive and radiological effects of radiotherapy in patients with low-grade glioma: long-term follow-up. Lancet Neurol 8(9):810-8.

17. Jolles J, Van Boxtel MP, Ponds RW, Metsemakers JF, Houx PJ (1998) [The Maastricht aging study (MAAS). The longitudinal perspective of cognitive aging]. Tijdschr Gerontol Geriatr 29(3):1209.

18. De Bie SE (1987) [Proposal for uniformisation of questions regarding background variables and interviews]. Leiden: Leiden University Press.

19. Lezak MD, Howieson DB, Loring DW (2004) Neuropsychological Assessment. New York: Oxford Univerity Press.

20. De Witt Hamer PC, Hendriks EJ, Mandonnet E, Barkhof F, Zwinderman AH, Duffau H (2013) Resection probability maps for quality assessment of glioma surgery without brain location bias. PLoS One 8(9):e73353.

21. Fonov V, McKinstry R, Almli C, Collins D (2009) Unbiased nonlinear average age-appropriate brain templates from birth to adulthood. Neuroimage 47:S102. 
22. Avants BB, Epstein CL, Grossman M, Gee JC (2008) Symmetric diffeomorphic image registration with cross-correlation: evaluating automated labeling of elderly and neurodegenerative brain. Med Image Anal 12(1):26-41.

23. Nichols TE, Holmes AP (2002) Nonparametric permutation tests for functional neuroimaging: a primer with examples. Hum Brain Mapp 15(1):1-25.

24. Nichols T, Hayasaka S (2003) Controlling the familywise error rate in functional neuroimaging: a comparative review. Stat Methods Med Res 12(5):419-46.

25. Schwartzman A, Dougherty RF, Lee J, Ghahremani D, Taylor JE (2009) Empirical null and false discovery rate analysis in neuroimaging. Neuroimage 44(1):71-82.

26. Desikan RS, Ségonne F, Fischl B, Quinn BT, Dickerson BC, Blacker D, et al (2006) An automated labeling system for subdividing the human cerebral cortex on MRI scans into gyral based regions of interest. Neuroimage 31(3):968-80.

27. Catani M, Thiebaut de Schotten M (2008) A diffusion tensor imaging tractography atlas for virtual in vivo dissections. Cortex 44(8):1105-32.

28. Team RC (2013) R: A Language and Environment for Statistical Computing. Vienna, Austria: R Foundation for Statistical Computing.

29. Gleichgerrcht E, Fridriksson J, Bonilha L (2015) Neuroanatomical foundations of naming impairments across different neurologic conditions. Neurology 85(3):284-92.

30. Dick AS, Tremblay P (2012) Beyond the arcuate fasciculus: consensus and controversy in the connectional anatomy of language. Brain 135(Pt 12):3529-50.

31. Petersen SE, Posner MI (2012) The attention system of the human brain: 20 years after. Annu Rev Neurosci 35:73-89.

32. Posner MI, Petersen SE (1990) The attention system of the human brain. Annu Rev Neurosci $13: 25-42$. 
33. Corbetta M, Shulman GL (2002) Control of goal-directed and stimulus-driven attention in the brain. Nat Rev Neurosci 3(3):201-15.

34. Posner MI, Sheese BE, Odludas Y, Tang Y (2006) Analyzing and shaping human attentional networks. Neural Netw 19(9):1422-9.

35. Rinne P, Hassan M, Goniotakis D, Chohan K, Sharma P, Langdon D, et al (2013) Triple dissociation of attention networks in stroke according to lesion location. Neurology 81(9):812-20.

36. Raz A (2004) Anatomy of attentional networks. Anat Rec B New Anat 281(1):21-36.

37. Corbetta M, Ramsey L, Callejas A, Baldassarre A, Hacker CD, Siegel JS, et al (2015) Common behavioral clusters and subcortical anatomy in stroke. Neuron 85(5):927-41.

38. Murakami T, Hama S, Yamashita H, Onoda K, Hibino S, Sato H, et al (2014) Neuroanatomic pathway associated with attentional deficits after stroke. Brain Res 1544:25-32.

39. Chiang HL, Chen YJ, Shang CY, Tseng WY, Gau SS (2016) Different neural substrates for executive functions in youths with ADHD: a diffusion spectrum imaging tractography study. Psychol Med 46(6):1225-38.

40. Ivanova MV, Dragoy O, Kuptsova SV, Yu Akinina S, Petrushevskii AG, Fedina ON, et al (2018) Neural mechanisms of two different verbal working memory tasks: A VLSM study. Neuropsychologia $115: 25-41$.

41. Owen AM, McMillan KM, Laird AR, Bullmore E (2005) N-back working memory paradigm: a meta-analysis of normative functional neuroimaging studies. Hum Brain Mapp 25(1):46-59.

42. Palacios EM, Sala-Llonch R, Junque C, Roig T, Tormos JM, Bargallo N, et al (2012) White matter integrity related to functional working memory networks in traumatic brain injury. Neurology 78(12):852-60.

43. Noll KR, Sullaway C, Ziu M, Weinberg JS, Wefel JS (2015) Relationships between tumor grade and neurocognitive functioning in patients with glioma of the left temporal lobe prior to surgical resection. Neuro Oncol 17(4):580-7. 
44. Karnath HO, Steinbach JP (2011) Do brain tumours allow valid conclusions on the localisation of human brain functions?--Objections. Cortex 47(8):1004-6.

45. Duffau H (2011) Do brain tumours allow valid conclusions on the localisation of human brain functions? Cortex 47(8):1016-7.

46. Kesler SR, Noll K, Cahill DP, Rao G, Wefel JS (2017) The effect of IDH1 mutation on the structural connectome in malignant astrocytoma. J Neurooncol 131(3):565-74.

47. Duffau H, Capelle L, Denvil D, Sichez N, Gatignol P, Lopes M, et al (2003) Functional recovery after surgical resection of low grade gliomas in eloquent brain: hypothesis of brain compensation. $\mathrm{J}$ Neurol Neurosurg Psychiatry 74(7):901-7.

48. Charras P, Herbet G, Deverdun J, de Champfleur NM, Duffau H, Bartolomeo P, et al (2015) Functional reorganization of the attentional networks in low-grade glioma patients: a longitudinal study. Cortex 63:27-41.

49. Visser M, Müller DMJ, van Duijn RJM, Smits M, Verburg N, Hendriks EJ, et al (2019) Inter-rater agreement in glioma segmentations on longitudinal MRI. Neuroimage Clin 22:101727.

50. Klein M, Engelberts NH, van der Ploeg HM, Kasteleijn-Nolst Trenite DG, Aaronson NK, Taphoorn MJ, et al (2003) Epilepsy in low-grade gliomas: the impact on cognitive function and quality of life. Ann Neurol 54(4):514-20.

51. Campanella F, Shallice T, Ius T, Fabbro F, Skrap M (2014) Impact of brain tumour location on emotion and personality: a voxel-based lesion-symptom mapping study on mentalization processes. Brain 137(Pt 9):2532-45.

52. Van Coevorden-van Loon EMP, Coomans MB, Heijenbrok-Kal MH, Ribbers GM. van den Bent MJ (2017) Fatigue in patients with low grade glioma: systematic evaluation of assessment and prevalence. J Neurooncol. 133(2):237-246. doi: 10.1007/s11060-017-2454-4. Epub 2017 May 24. 53. Talacchi A, Santini B, Savazzi S, Gerosa M (2011) Cognitive effects of tumour and surgical treatment in glioma patients. J Neurooncol 103(3):541-549

54. Merkley TL, Larson MJ, Bigler ED, Good DA, Perlstein WM (2013) Structural and functional changes of the cingulate gyrus following traumatic brain injury: relation to attention and executive skills. J Int Neuropsychol Soc. 19(8):899-910. doi: 10.1017/S135561771300074X. 
Table 1. Sociodemographic and clinical characteristics of the patient cohort

\begin{tabular}{|c|c|}
\hline & No. of patients $(n=72)$ \\
\hline $\mathrm{Age}^{1} ;$ mean $\pm \mathrm{SD}$ & $39.8 \pm 12.3$ \\
\hline \multicolumn{2}{|l|}{ Sex No. $(\%)$} \\
\hline Male & $45(63 \%)$ \\
\hline Educational level $^{2}$; mean \pm SD & $4.5 \pm 1.9$ \\
\hline \multicolumn{2}{|l|}{ Glioma WHO grade } \\
\hline $\mathrm{I}$ & $7(10 \%)$ \\
\hline II & $43(60 \%)$ \\
\hline III & $12(16 \%)$ \\
\hline IV & $10(14 \%)$ \\
\hline Tumor location; left No. (\%) & $41(57 \%)$ \\
\hline \multicolumn{2}{|l|}{ Presenting symptom(s) No. (\%) } \\
\hline Epilepsy & $45(62 \%)$ \\
\hline Epilepsy and neurocognitive impairment & $7(10 \%)$ \\
\hline Epilepsy and neurological impairment & $5(7 \%)$ \\
\hline Neurocognitive deficits & $4(6 \%)$ \\
\hline Neurological impairment & $5(7 \%)$ \\
\hline Headache & $1(1 \%)$ \\
\hline None & $5(7 \%)$ \\
\hline Use of AEDs No. (\%) & $48(67 \%)$ \\
\hline Use of corticosteroids No. (\%) & $6(8 \%)$ \\
\hline \multicolumn{2}{|c|}{$\begin{array}{l}\text { Interval between neuropsychological examination } \\
\text { and preoperative } \mathrm{MRI}^{4} \text {; }\end{array}$} \\
\hline - mean $\pm \mathrm{SD} /$ median & $0.4 \pm 9.2 / 0$ \\
\hline - range & $0-35.6$ \\
\hline
\end{tabular}

${ }^{1}$ Years; ${ }^{2}$ Code $1-8$, ranging from lower general education to university education $[18] ;{ }^{3}$ Larges diameter in mm on MRI T1 with contrast for grade III-IV, and on MRI FLAIR for grade I and II; ${ }^{4}$ Weeks; AEDs: anti-epileptic drugs 


\section{Tables and figures}

Table 1. Sociodemographic and clinical characteristics of the patient cohort

${ }^{1}$ Years; ${ }^{2}$ Code $1-8$, ranging from lower general education to university education(18); ${ }^{3}$ Larges diameter in mm on MRI T1 with contrast for grade III-IV, and on MRI FLAIR for grade I and II; ${ }^{4}$ Weeks

Fig. 1 Glioma locations within the brain

Transversal sections are shown superimposed on standard brain space (MNI). The legend refers to the number of patients with glioma tissue at a voxel, with lighter yellow indicating a higher number of patients.

Fig. 2 Distribution of neurocognitive functioning scores per domain $(\mathrm{n}=72)$

Each bar represents one patient, with darker colors indicating scores below $1.5 \mathrm{SD}$ of healthy controls (horizontal dotted line at $-1.5 \mathrm{SD}$ ).

Fig. 3 Tumor localization map for the attention and working memory domains

Fig. 3a TLM of (A) patients with a tumor at that voxel without an attention deficit, and of (B) patients with a tumor and an attention deficit. Lighter yellow indicates higher percentages. (C) Relative risk map of tumor probability, i.e. the odds ratio at each voxel between tumor probabilities (A) and (B), with darker green indicating more deficits in presence of a tumor. The observed relative differences were voxel-wise tested for significance, resulting in an adjusted p-value map (D), taking spatial dependence into account. Lighter yellow indicates lower p-values. The corrected q-value map to address multiple testing (E), displayed as the log odds ratios for the voxels with q-values below 0.4 presented in red and q-values below 0.2 in yellow. Voxel clusters with $\mathrm{q}<0.4$ were considered significant.

Fig. $3 b$ Tumor localization map for the working memory domain

For a detailed legend, see figure $3 \mathrm{a}$.

Fig. 4 Heat map with the association between anatomical brain structures and neurocognitive domains The neurocognitive domains are shown in columns, grouped per hemisphere and the midline. Anatomical structures are displayed in rows, grouped per cortical region, subcortical white matter structure and subcortical nucleus. Horizontal orientation shows the percentage of significant voxels (with $\mathrm{q}<0.4$ ) per domain of the total number of significant voxels per anatomical structure, with darker shades of green indicating a stronger association. 


\section{Electronic Supplementary Material}

Online recourse 1: Table 1 Cognitive domains and tests

* higher score means better performance; \# higher score means worse performance

Online resource 2: Video 1 Axial sections of tumor-infiltrated brain regions associated with language dysfunction.

The results identify established language regions. Results are superimposed on MNI standard brain template: (1) tumor map of 46 patients without language dysfunction, (2) tumor map of 13 patients with language dysfunction, (3) relative risk map of tumor location with and without language dysfunction, (4) p-value map of randomization tests, (5) q-value map of false discovery rate. The numbers indicate MNI z-values.

Online resource 3: Video 2 Axial sections of tumor regions associated with attention impairment. Results are superimposed on MNI standard brain template: (1) lesion map of regions of 49 patients without impairment, (2) lesion map of 22 patients with attention impairment, (3) relative risk map of regions with and without attention impairment, (4) p-value map of randomization tests, (5) q-value map of false discovery rate. The numbers indicate MNI z-values.

Online resource 4: Video 3 Axial sections of tumor regions associated with working memory impairment. Results are superimposed on MNI standard brain template: (1) lesion map of regions of 57 patients without impairment, (2) lesion map of 14 patients with working memory impairment, (3) relative risk map of regions with and without working memory impairment, (4) p-value map of randomization tests, (5) q-value map of false discovery rate. The numbers indicate MNI z-values.

Online resource 5: Video 4 Axial sections of tumor regions associated with visual memory impairment. Results are superimposed on MNI standard brain template: (1) lesion map of regions of 60 patients without impairment, (2) lesion map of 10 patients with visual memory impairment, (3) relative risk map of regions with and without visual memory impairment, (4) p-value map of randomization tests, (5) q-value map of false discovery rate. The numbers indicate MNI z-values.

Online resource 6: Video 5 Axial sections of tumor regions associated with verbal memory impairment. Results are superimposed on MNI standard brain template: (1) lesion map of regions of 62 patients without impairment, (2) lesion map of 9 patients with verbal memory impairment, (3) relative risk map of regions with and without verbal memory impairment, (4) p-value map of randomization tests, (5) q-value map of false discovery rate. The numbers indicate MNI z-values.

Online resource 7: Video 6 Axial sections of tumor regions associated with information processing speed impairment.

Results are superimposed on MNI standard brain template: (1) lesion map of regions of 63 patients without impairment, (2) lesion map of 8 patients with information processing speed impairment, (3) relative risk map of regions with and without information processing speed impairment, (4) p-value map of randomization tests, (5) q-value map of false discovery rate. The numbers indicate MNI z-values.

Online resource 8: Video 7 Axial sections of tumor regions associated with impairment in executive functioning.

Results are superimposed on MNI standard brain template: (1) lesion map of regions of 65 patients without impairment, (2) lesion map of 6 patients with executive functioning impairment, (3) relative risk map of regions 
Habets et al. 20

with and impairment in executive functioning, (4) p-value map of randomization tests, (5) q-value map of false discovery rate. The numbers indicate MNI z-values. 